Rodrigo Borges da Silva Santos

\title{
Sistema de Controle de Versões para Edição Cooperativa de Vídeo \\ MPEG-2
}

DissertaçÃo de Mestrado

DEPARTAMENTO DE INFORMÁTICA

Programa de Pós-Graduação em Informática 


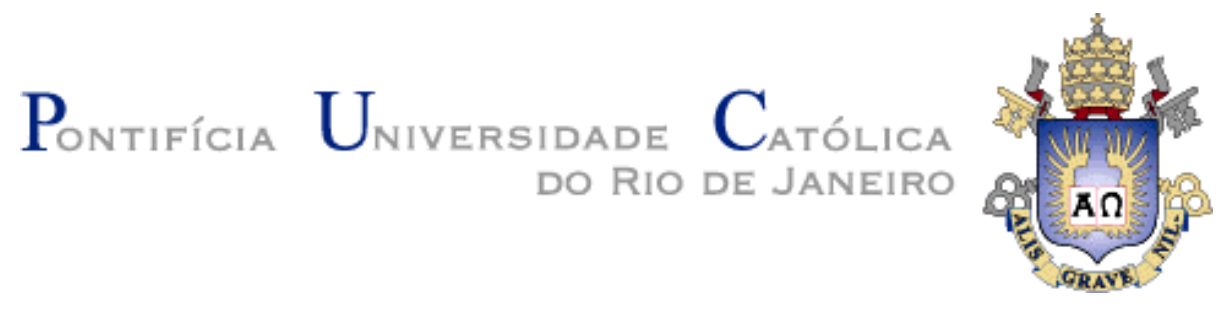

Rodrigo Borges da Silva Santos

Sistema de Controle de Versões para Edição Cooperativa de Vídeo MPEG-2

Dissertação de Mestrado

Dissertação apresentada como requisito parcial para obtenção do título de Mestre pelo Programa de PósGraduação em Informática da PUC-Rio.

Orientador: Luiz Fernando Gomes Soares Co-orientador: Marco Antônio Casanova

Rio de Janeiro, 15 de março de 2007 


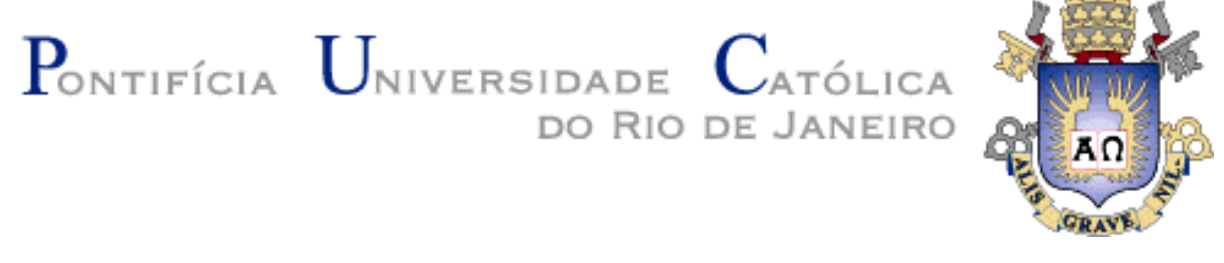

Rodrigo Borges da Silva Santos

\section{Sistema de Controle de Versões para Edição Cooperativa de Vídeo MPEG-2}

Dissertação apresentada como requisito parcial para obtenção do título de Mestre pelo Programa de PósGraduação em Informática da PUC-Rio. Aprovada pela Comissão Examinadora abaixo assinada.

Luiz Fernando Gomes Soares Orientador Departamento de Informática - PUC-Rio

Marco Antonio Casanova Co-Orientador Departamento de Informática - PUC-Rio

Bruno Feijó Departamento de Informática - PUC-Rio

Rogério Ferreira Rodrigues Departamento de Informática - PUC-Rio

José Eugênio Leal

Coordenador Setorial do Centro Técnico Científico - PUC-Rio

Rio de Janeiro, 15 de março de 2007. 
Todos os direitos reservados. É proibida a reprodução total ou parcial do trabalho sem autorização da universidade, do autor e do orientador.

\section{Rodrigo Borges da Silva Santos}

Bacharel em Ciência da Computação graduado pela Universidade Federal de Pernambuco (UFPE) em outubro de 2003.

Ficha Catalográfica

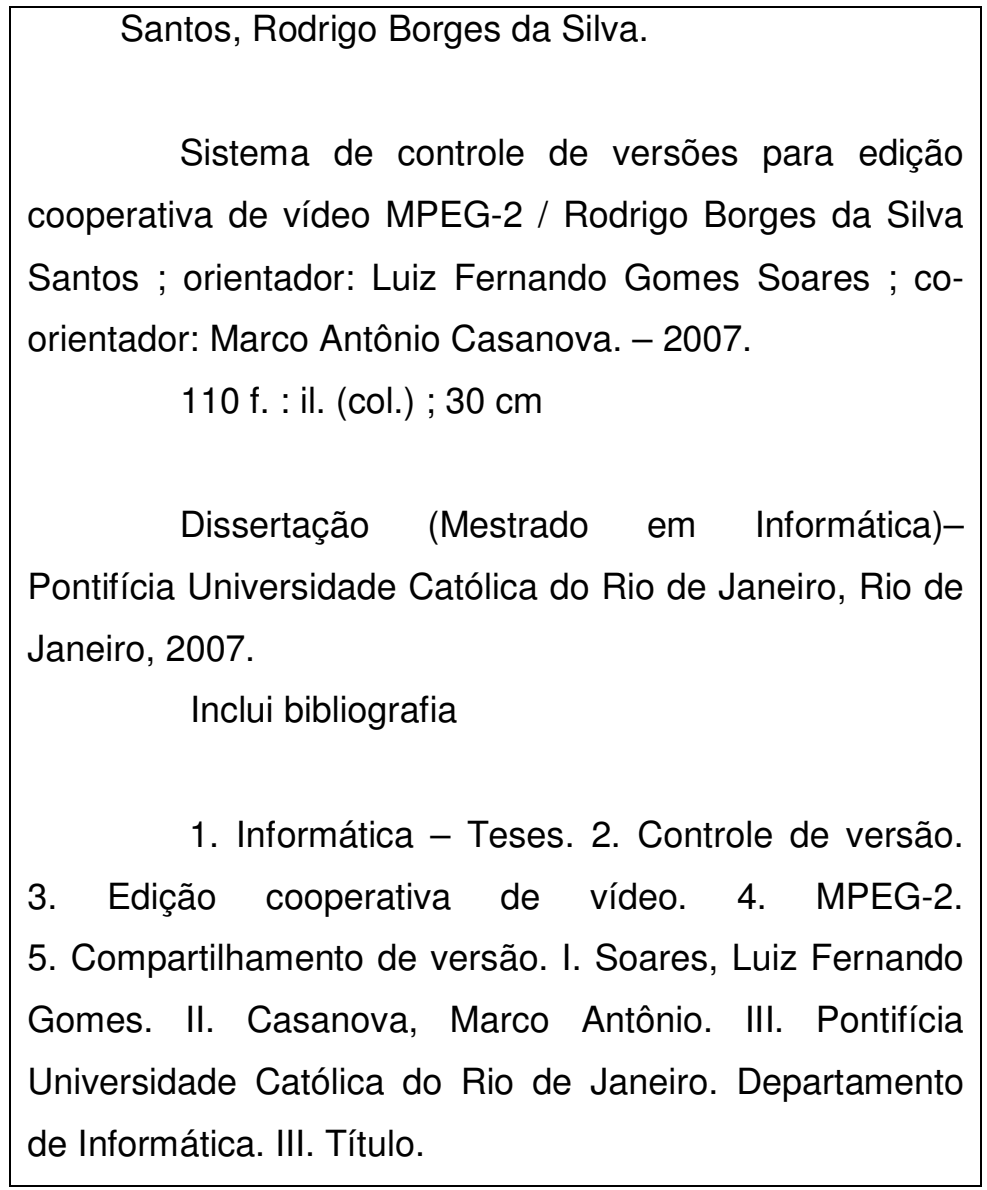

CDD: 004 
Este trabalho é dedicado

aos meus pais, Edmilson e Antônia, e as minhas irmãs, Renata e Rafaella, por todo amor que dedicam a nossa família.

À Patrícia, que mesmo distante, é minha inseparável companheira, fonte de amor e de minha alegria. 


\section{Agradecimentos}

Aos meus orientadores, Professor Luiz Fernando e Marco Antonio Casanova, por terem me orientado com paciência e respeito, sempre solícitos em ouvir minhas dúvidas e inquietações.

À minha família, pelo apoio e incentivo demonstrados em todos os momentos de minha vida.

À Patrícia, por seu amor, carinho e compreensão.

À equipe do Laboratório TeleMídia, pela amizade e companheirismo, que muito contribuíram para a realização deste trabalho de uma forma mais prazerosa. Em especial, a Macarrão (vulgo Laiola) e Lambão (vulgo Rafael Rodrigues), pela amizade, presença constante, apoio e orientação indispensáveis à efetivação deste trabalho.

A todos os meus amigos e primos pelo carinho e confiança. Aos companheiros de longa data, amigos de Pernambuco, em especial Moacir, Zeca, Katyusco e Lulu. Também aos amigos Malcher, Renato e Vinicius.

Aos membros da banca pelos comentários pertinentes e pelas revisões precisas. Agradeço também aos professores e funcionários do Departamento de Informática da PUC-Rio que colaboraram para a conclusão deste trabalho.

À CNPQ pelo apoio financeiro que proporcionou a realização deste trabalho. 


\section{Resumo}

Santos, Rodrigo Borges da Silva; Soares, Luiz Gomes Soares (orientador); Casanova, Marco Antônio (co-orientador). Sistema de Controle de Versões para Edição Cooperativa de Vídeo MPEG-2. Rio de Janeiro, 2007. 110p. Dissertação de Mestrado - Departamento de Informática, Pontifícia Universidade Católica do Rio de Janeiro.

Os avanços das tecnologias de captura, armazenamento e compressão de vídeo digital estão motivando o desenvolvimento e a disponibilização de novos serviços e sistemas para manipulação e gerenciamento de acervos de vídeo. Um exemplo disso são os sistemas de gerenciamento, edição e compartilhamento de versões utilizados pelos produtores de conteúdo audiovisual. Entretanto, tais funcionalidades são requisitos não encontrados em um único sistema. Este trabalho descreve um sistema que possibilita a edição cooperativa de dados audiovisuais no formato MPEG-2 permitindo o controle de versão, a visualização e manipulação do seu conteúdo por partes (segmentos). Esse sistema colaborativo tem ainda como vantagens a divisão de tarefas, a fusão das contribuições e a extração de informações da autoria de cada versão.

\section{Palavras-chave}

Controle de Versão; Edição Cooperativa de Vídeo; MPEG-2; Compartilhamento de Versão. 


\section{Abstract}

Santos, Rodrigo Borges da Silva; Soares, Luiz Fernando Gomes (advisor); Casanova, Marco Antônio (co-advisor). Version Control System for Cooperative MPEG-2 Video Editing. Rio de Janeiro, 2007. 100p. Master Thesis - Departamento de Informática, Pontifícia Universidade Católica do Rio de Janeiro.

Technological advances in areas such as capture, storage and compression of digital video are stimulating the development of new services and systems for manipulation and management of huge amount of video data. An example of this, are the systems of management, editing and sharing of versions used by producers of audiovisual content. However, such functional requirements are not found in one system. This work describes a system that makes possible the cooperative edition of audiovisual data in MPEG-2 format, allowing the version control, visualization and manipulation of its content by segments. This collaborative system still has advantages as the division of tasks between editors, the fusion of different versions and the extraction of information of authorship from each version.

\section{Key words}

Version Control; Cooperative Video Editing; MPEG-2; Version Sharing. 


\section{Sumário}

1 Introdução 13

1.1. Motivação 13

1.2. Objetivos 16

$\begin{array}{ll}\text { 1.3. Estrutura da Dissertação } & 17\end{array}$

2 Conceitos Gerais do Padrão MPEG-2 18

2.1. O Fluxo MPEG-2 de Sistemas 19

2.2. O Fluxo MPEG-2 de Vídeo 21

2.2.1. Estruturas de um fluxo de vídeo MPEG-2 21

2.2.2. Controle de ocupação do buffer 25

3 Trabalhos Relacionados 30

3.1. Sistemas de Controle de Versão 30

3.1.1. Conceitos básicos 31

3.1.2. Envio e resgate de versões 33

3.1.3. Trabalho Colaborativo 34

3.2. Sistemas de Edição de Vídeo Colaborativo 36

3.2.1. Mecanismos de Segmentação e Remontagem do MPEG 36

3.2.2. Ferramentas de Edição de Vídeo Colaborativo 38

4 Modelo de Dados do Controle de Versões para Edição Cooperativa de Vídeo

4.1. Árvore de Versionamento de um Vídeo 42

4.2. Acessos Concorrentes aos Nós 45

4.2.1. Versão Temporária e Permanente da Árvore de Versionamento 50

4.3. Granularidade Múltipla na Árvore de Versionamento 52

4.3.1. Bloqueios Explícitos e Implícitos no modo Exclusivo ou

Compartilhados 53

4.3.2. Bloqueios Intencionais no modo Exclusivo ou Compartilhado 54

4.3.3. Protocolo de Bloqueio em Duas Fases 56 
4.3.4. Nós Compartilhados entre Árvores de Versionamento 58

4.4. Mecanismo de Segmentação 60

4.5. Mecanismo de Remontagem 63

4.6. Fusão entre Árvores de Versionamento 64

5 Implementação 68

5.1. Desenvolvimento do VideoCVS 68

5.2. Arquitetura do VideoCVS 69

5.3. Modelo Entidade e Relacionamento do VideoCVS 75

5.3.1. Classes dos Objetos Remotos 76

5.3.2. Classes do Repositório 78

5.4. Mecanismo de Segmentação 79

5.5. Mecanismo de Remontagem 81

5.6. Exemplo de Uso do VideoCVS 82

5.6.1. Criação de uma Árvore de Versionamento 82

5.6.2. Checkout de uma Árvore de Versionamento 86

5.6.3. Edição Colaborativa de uma Árvore de Versionamento 87

5.6.4. Fusão entre Árvores de Versionamento 91

5.6.5. Remontagem dos Segmentos da Árvore de Versionamento 94

6 Conclusão 96

6.1. Contribuições da Dissertação 96

6.2. Trabalhos Futuros $\quad 97$

$\begin{array}{ll}\text { Referências } & 101\end{array}$

$\begin{array}{ll}\text { Anexo A } & 105\end{array}$ 


\section{Lista de figuras}

Figura 1 - Efeito do processo de decodificação e codificação em cascata........... 15

Figura 2 - Estrutura do fluxo MPEG-2 ........................................................ 19

Figura 3 - Sincronização entre o codificador e o decodificador ..........................220

Figura 4 - Estrutura do fluxo MPEG Vídeo ................................................. 21

Figura 5 - Ordem de apresentação e codificação dos quadros..............................25

Figura 6 - Exemplo de múltiplas versões organizadas em revisões..................... 32

Figura 7 - Histórico de versões no sistema estilo CVS...................................... 34

Figura 8 - Histórico de versões no sistema estilo SVN.................................... 34

Figura 9 - Detecção das Tomadas de Cenas de um Noticiário de Telejornal....... 40

Figura 10 - Exemplo de uma árvore de versionamento de vídeo ........................43

Figura 11 - Compartilhamento de nós entre árvores de versionamento................45

Figura 12 - Problema do acesso simultâneo de um nó..........................................46

Figura 13 - Método Lock-Modify-Unlock ou Exclusive Lock.............................. 47

Figura 14 - Método Copy-Modify-Merge ou Optimistic Merges......................... 48

Figura 15 - Continuação do exemplo do método copy-modify-merge ................. 49

Figura 16 - Versão permanente e temporária .................................................50

Figura 17 - Versão temporária editada por um único usuário .............................51

Figura 18 - Versão temporária editada por vários usuários.................................52

Figura 19 - Árvore de versionamento com bloqueio X e S ..............................53

Figura 20 - Árvore de versionamento com bloqueio intencional .........................55

Figura 21 - Exemplo de bloqueio de um nó no modo exclusivo ..........................59

Figura 22 - Estado de bloqueio dos nós .......................................................... 60

Figura 23 - Fases do mecanismo de segmentação ............................................. 61

Figura 24 - Tratamento das bordas dos segmentos .........................................63

Figura 25 - Detecção das tomadas de cenas de um vídeo .................................63

Figura 26 - Fusão de duas árvores de versionamento ........................................66

Figura 27 - Fusão das árvores de versionamento com intervenção do usuário ....66

Figura 28 - Fusão de duas árvores de versionamento com nós não equivalentes 67

Figura 29 - Fusão de duas árvores de versionamento com nós invertidos 67 


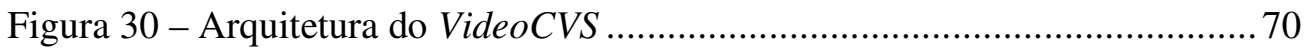

Figura 31 - Diagrama de classes do VideoCVS Server..................................... 71

Figura 32 - Exemplo de commit na arquitetura do VideoCVS.......................... 73

Figura 33 - Diagrama de classes do VideoCVS Client ...................................... 74

Figura 34 - Modelo ER do sistema VideoCVS................................................ 75

Figura 35 - Diagrama de classes dos objetos remotos do modelo ER ................ 77

Figura 36 - Diagrama de classes dos objetos remotos do repositório .................. 78

Figura 37 - Diagrama de classes do mecanismo de segmentação ....................... 79

Figura 38 - Diagrama de classes da estrutura do MPEG-2 de vídeo ................... 80

Figura 39 - Diagrama de classes do mecanismo de remontagem ....................... 81

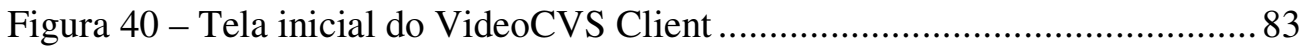

Figura 41 - Primeira tela da criação da árvore de versionamento........................ 84

Figura 42 - Segunda tela da criação da árvore de versionamento........................ 84

Figura 43 - Quadros iniciais dos segmentos do vídeo pinpong. $m 2 v$................... 85

Figura 44 - Commit da árvore de versionamento pelo usuário 1 ........................ 85

Figura 45 - Operação de checkout de uma árvore de versionamento .................. 86

Figura 46 - Resultado da busca do checkout ................................................... 87

Figura 47 - Bloqueio no modo exclusivo permitido ao usuário 1 ..........................8 88

Figura 48 - Bloqueio no modo exclusivo negado ao usuário 2 …..................... 88

Figura 49 - Bloqueio no modo compartilhado negado ao usuário 2 .................. 89

Figura 50 - Clicando na opção Preview para visualizar o vídeo ........................ 89

Figura 51 - Bloqueios no modo exclusivo permitido ao usuário 2 …..................90 90

Figura 52 - Desbloqueio de um nó da árvore de versionamento pelo usuário 2 .. 90

Figura 53 - Permissão negada para bloquear na fase de encolhimento................ 91

Figura 54 - Merge entre versões .................................................................. 92

Figura 55 - Fusão entre as árvores de versionamento tree_v12 e tree_v13......... 92

Figura 56 - Intervenção manual do usuário na fusão entre tree_v13 e tree_v14..93

Figura 57 - Árvores de versionamento resultantes após intervenção do usuário.. 93

Figura 58 - Operação de inserção de nós na árvore de versionamento ................. 94

Figura 59 - Árvore de versionamento tree_v2 para remontagem ....................... 95

Figura 60 - Preview da remontagem da tree_v2 ........................................... 95 


\section{Lista de tabelas}

Tabela 1 - Parâmetros repeat_first_field e top_field_first..................................22

Tabela 2 - Resumo da sintaxe das camadas do MPEG-2 de Vídeo .....................24

Tabela 3 - Matriz de compatibilidade dos modos de bloqueio ............................56

Tabela 4 - Exemplo usando o protocolo de bloqueio em duas fases ...................57 